\title{
A Facile Scalable Conductive Graphene-Coated Calotropis Gigantea Yarn
}

Junze Zhang

Soochow University

Jing Liu

Soochow University

Zeyu Zhao

Soochow University

Di Huang

Soochow University

Chao Chen

Soochow University

Zhaozhu Zheng

Soochow University

Chenxi Fu

Soochow University

Xiaoqin Wang

Soochow University

Yanxue Ma

Donghua University

Yuling Li

Donghua University

Zekun Liu ( $\nabla$ zekun.liu@manchester.ac.uk)

The University of Manchester

Yi Li

The University of Manchester

Gang Li

Soochow University

\section{Research Article}

Keywords: Graphene, Calotropis gigantea, Conductive fibers, Coating, Wearable electronic devices

Posted Date: September 24th, 2021 
DOI: https://doi.org/10.21203/rs.3.rs-861664/v1

License: (c) (1) This work is licensed under a Creative Commons Attribution 4.0 International License. Read Full License

Version of Record: A version of this preprint was published at Cellulose on March 1st, 2022. See the published version at https://doi.org/10.1007/s10570-022-04475-z. 


\section{Abstract}

Graphene-functionalized fibers have attracted substantial attention due to their potential applications in flexible wearable electronic devices. However, these conventional conductive materials are facing difficulties in mass production. In this paper, we report a graphene-coated Calotropis gigantea yarn by pad dyeing with graphene oxide and reduction process, which endows it with high conductivity, outstanding conducting stability, and scale production capacity. By optimizing the dyeing parameters, the modified Calotropis gigantea yarns display high electrical conductivity of $6.9 \mathrm{~S} / \mathrm{m}$. Range analysis results indicate that electrical conductivity of the graphene-coated yarns exhibits strong dependence on the concentration of graphene oxide and pad dyeing cycles. The combination between hydrogen bond of the fiber and the oxygen bond of graphene during dyeing process renders the functionalized yarns stable conductivity to washing and bending. Based on the simple fabrication process, and fascinating performance, the graphene-coated yarns show great potential in facile scale production.

\section{Introduction}

In recent years, the explosion of multi-functional flexible wearable electronic textiles in flexible sensors ${ }^{[1-}$ ${ }^{5]}$, wearable electronic devices ${ }^{[6-8]}$, electromagnetic interference shielding ${ }^{[9-11]}$ has attracted extensive attention due to their great potential as next-generation electronics with the advantages of lightweight, flexibility, and portability, which can be applied for personal motion detection and healthcare, manmachine interaction, artificial intelligence and so forth ${ }^{[12]}$. There are many approaches to prepare electronic fibers through the modification of polyaniline, metal nanoparticles, carbon nanotubes, graphene etc. Graphene possesses a unique 2-D sheet-like structure ${ }^{[13]}$, with extremely high electrical conductivity, high mechanical strength, and good chemical stability ${ }^{[14]}$. Compared with other materials, graphene can be much easier to cover along the fiber surface under shearing force, which can form a full coverage on the fiber surface. On the other hand, strong $\pi-\pi$ interactions existing between adjacent graphene are beneficial to forming interconnected electron-transport pathways for high electrical conductivity ${ }^{[15]}$.

Calotropis gigantea yarns (CGYs) have received intense attention in recent years due to its unique structure and performance ${ }^{[16]}$. Calotropis gigantea fibers (CGFs) with $80-90 \%$ hollow structures exhibit outstanding hydrophilic or oleophilic properties ${ }^{[17,18]}$. Compared to cotton fibers, CGFs with lightweight have fewer natural curls in the longitudinal direction, resulting in a smoother and softer surface. By taking advantages of the low cost, low density, environmental friendliness, and high specific mechanical properties of CGFs, composites made with the natural cellulose fiber were investigated for the applications of reducing the wear of carbon nanotube-based composites, improving the mechanical strength of polyester-based composites ${ }^{[19,20]}$. Even though these studies focused on the properties of the CGF-based composites, the functionalization of CGFs by the coating of graphene was not investigated. More importantly, scale production of such graphene-coated CGFs has always been a great challenge, which has not been reported. 
Herein, we report a graphene-coated CGY by pad dyeing, which endows it with not only high performance in conductivity but also scale capacity in production. By optimizing the dyeing parameters such as graphene oxide concentration, pad dyeing cycles, and reduction time, the graphene-coated CGYs display high electrical conductivity of $6.9 \mathrm{~S} / \mathrm{m}$. We also compare the dyeing parameters in determining the conductivity of the yarns through range analysis, showing that the electrical conductivity of the graphenecoated CGYs exhibits strong dependence on the concentration of graphene oxide and pad dyeing cycles. The combination between hydrogen bond of CGFs and the oxygen bond of graphene renders the modified CGYs good conducting stability to washing and bending. Based on the advantages of simple process, outstanding conductivity, and stability, the graphene-coated CGYs with mass production capacity show great potential in the application of wearable textile-based electronics.

\section{Experimental}

\subsection{Materials}

Graphene oxide (GO) solution was prepared by Hummers method using natural graphite from Suzhou Industrial Park Bomeida Reagent Instrument Co., Ltd as precursors. The CGYs were supplied from Yixing zhongchang yarn-dyed Co., Ltd. Analytical-grade reagents of sodium, hydrochloric acid, potassium permanganate $\left(\mathrm{KMnO}_{4}\right)$ were purchased from Sinopharm Chemical Reagent Co., Ltd, China.

Concentrated sulfuric acid, hydrogen peroxide with $30 \%$ concentration, and Ascorbic acid were from Jiangsu Qiangsheng Functional Chemical Co., Ltd, China, Shanghai Lingfeng Chemical Reagent Co., Ltd, China, and Suzhou Ketong Biomedical Technology Co., Ltd, China respectively.

\subsection{Preparation of graphene coated CGYs}

GO solution was synthesized by Hummers method, the synthesis approach was also reported in our previous work ${ }^{[21]}$. Firstly, mixing graphite powders $(2 \mathrm{~g}), \mathrm{NaNO}_{3}(2 \mathrm{~g})$, and $50 \mathrm{~mL}$ of $\mathrm{H}_{2} \mathrm{SO}_{4}(98 \%)$ in a $1000 \mathrm{~mL}$ volumetric flask and keeping them in an ice bath. The mixture was constantly stirred for $2 \mathrm{~h}$ with the gradual addition of potassium permanganate $(6 \mathrm{~g})$. Then, removing the ice bath and stirring the mixture at $35^{\circ} \mathrm{C}$ until it became pasty brownish and kept under stirring for 2 days. The mixture was diluted with distilled water, and then $10 \mathrm{~mL} \mathrm{H}_{2} \mathrm{O}_{2}$ was added to terminate the reaction. For purification, we washed the mixture by rinsing and centrifugation with $10 \% \mathrm{HCl}$ and then deionized (DI) water several times. As a result, GO solution with uniform dispersion was obtained. In order to form a high-quality coating on the surface of the CGYs. The CGYs were immersed in GO solution for $2 \mathrm{~h}$, then the CGYs passed through a small rolling mill at a speed of approximately $0.1 \mathrm{~m} / \mathrm{s}$ to remove excess $\mathrm{GO}$ on the surface. Subsequently, the coated yarns were dried in an oven at $60^{\circ} \mathrm{C}$ for $1 \mathrm{~h}$ to fix GO on the surface of the CGYs. By using green reducing agent ascorbic acid to reduce $\mathrm{GO}$ on the surface of pristine CGYs at the temperature of $80^{\circ} \mathrm{C}$ for $24 \mathrm{~h}$. After this step, the reduced GO ( $\mathrm{rGO}$ ) modified CGYs can be successfully obtained.

\subsection{Characterization}


We measured the contact angles by a contact angle measuring equipment (Krüss DSA 100, Germany) to characterize the wettability of the CGYs. Before measurement, arranged the CGYs on the glass slide, and the distance between adjacent CGYs is $0.28 \mathrm{~mm}$. The tensile properties of the CGYs were tested by a universal material testing machine (INSTRON-3365, Instron, USA). Electrical properties of the rGO-CGYs were measured using a Keithley DMM7510 instrument. Clamped the rGO-CGYs with copper wire at a distance of $5 \mathrm{~cm}(\mathrm{I})$, and conductive silver paste was used to fix and minimize contact resistance between samples and electrodes ${ }^{[22]}$. The thermogravimetric analysis (TGA) was performed on a SDT Q600 (simultaneous DSC-TG analyzer, TA Instruments, USA) to study the thermal stability of the CGYs. The surface morphologies and elemental distribution of the CGYs were measured using a scanning electron microscope (SEM, Hitachi TM3030, Tokyo, Japan). The secondary structure of the CGYs was examined using Fourier transform infrared spectroscopy (FT-IR) spectroscopy (Nicolet 5700, USA) to study the binding mechanism of the pristine CGY and graphene. Raman spectra of the CGYs were characterized on Jobin Yvon LabRam HR800, excited by $632.8 \mathrm{~nm}$ laser. We washed the rGO-CGY with deionized water and laundry liquid for different time to study the stability of the resistance.

\section{Results And Discussion}

\subsection{Graphene coating onto CGYs}

GO possesses good dispersion stability in solution, which is due to the strong electrostatic repulsion between the charged $\mathrm{GO}{ }^{[23]}$. Although $\mathrm{GO}$ can be easily attached onto CGYs by physical bonging, it is difficult to obtain a completely covered structure due to the intrinsic repulsion between $\mathrm{GO}$ and the coated yarns. We, therefore, employ a commercialized pad dyeing instrument to accomplish the modification. Figure 1a shows the detailed preparation procedure of rGO-CGYs. The pristine CGYs were firstly immersed into GO solution to make surface fully in contact with the GO solution. Then the excess GO solution on the pristine CGYs surface was removed by a rolling mill to obtain continuous structure and full coverage for high-efficiency electron transport. After the GO modification, the GO-coated yarn experienced reduction through being transferred into ascorbic acid solvent (with a weight ratio of $10: 1$ to $\mathrm{GO}$ ) at $80^{\circ} \mathrm{C}$ to acquire rGO-CGYs.

Figure $1 \mathrm{~b}$ presents the schematic diagrams of pristine CGY, GO-CGY, and $\mathrm{rGO}-\mathrm{CGY}$, and the photos of corresponding yarns. The size distribution of GO in Fig. 1c exhibits that the size of the $\mathrm{GO}$ flakes is mainly between $2 \mu \mathrm{m}$ and $6 \mu \mathrm{m}$ and we can see that the GO flakes possess a uniform size, which is beneficial to pad dyeing of CGYs. In order to optimize the experimental parameters, we analyze the GO concentration, pad dyeing cycles, and reduction time in determining the properties of graphene-coated CGYs through an orthogonal design with three factors and three levels. Table 1 shows the sample specifications. We choose the samples with good volume conductivity (Sample 2, Sample 5, and Sample 8) to present and evaluate their performance, which are denoted as rGO-CGY-4, rGO-CGY-7 and rGO-CGY-10 respectively. The factors affecting the yarn conductivity are analyzed using range analysis with all sample data 
included. Figure $1 \mathrm{~d}$ illustrates that the viscosity of $\mathrm{GO}$ solutions of 3,5 , and $7 \mathrm{mg} / \mathrm{ml}$ is $26.6 \pm 4.8,41.4 \pm$ 7.4 , and $61.6 \pm 7.0$ respectively, indicating a higher $\mathrm{GO}$ concentration obviously increases its viscosity.

Table 1

The specifications of rGO-CGYs through pad dyeing.

\begin{tabular}{|llllll|}
\hline Sample & $\begin{array}{l}\text { Additive } \\
\text { concentration } \\
(\mathrm{mg} / \mathrm{mL})\end{array}$ & $\begin{array}{l}\text { Reduction } \\
\text { time } \\
(\mathrm{h})\end{array}$ & $\begin{array}{l}\text { Pad dyeing } \\
\text { cycles }\end{array}$ & $\begin{array}{l}\text { Contact } \\
\text { angles } \\
\left({ }^{\circ}\right)\end{array}$ & $\begin{array}{l}\text { Volume } \\
\text { conductivities } \\
(\mathbf{S} / \mathrm{m})\end{array}$ \\
\hline 1 & 3 & 12 & 4 & $133.83 \pm 5.12$ & $0.29 \pm 0.08$ \\
\hline 2 & 5 & 36 & 4 & $147.47 \pm 5.21$ & $1.99 \pm 0.35$ \\
\hline 3 & 7 & 24 & 4 & $135.23 \pm 4.08$ & $0.56 \pm 0.12$ \\
\hline 4 & 3 & 24 & 7 & $139.03 \pm 8.49$ & $0.33 \pm 0.05$ \\
\hline 5 & 5 & 12 & 7 & $150.27 \pm 4.94$ & $3.50 \pm 0.44$ \\
\hline 6 & 7 & 36 & 7 & $145.03 \pm 2.97$ & $0.98 \pm 0.09$ \\
\hline 7 & 3 & 36 & 10 & $143.83 \pm 5.00$ & $0.74 \pm 0.05$ \\
\hline 8 & 5 & 24 & 10 & $150.1 \pm 3.52$ & $6.90 \pm 0.19$ \\
\hline 9 & 7 & 12 & 10 & $142.33 \pm 5.87$ & $1.40 \pm 0.22$ \\
\hline
\end{tabular}

\subsection{Surface morphology and elements}

The pristine CGY and rGO-CGY-10 in Fig. 2a, b show that the nature-color yarn becomes black after the functionalization, which can be attributed to the attachment of rGO onto the fiber surface. After the modification, the surface elements of the yarns are significantly changed. Figure $2 \mathrm{c}$ shows the atomic percentage of $\mathrm{C}, \mathrm{N}$ and $\mathrm{O}$ on the surface of pristine CGY, GO-CGY-10, and $\mathrm{rGO}-\mathrm{CGY}-10$. It presents that the atomic percentage of $\mathrm{C}, \mathrm{N}$, and $\mathrm{O}$ of the pristine yarn is $57.780 \%, 4.678 \%$, and $37.542 \%$ respectively, while that becomes $59.323 \%, 3.133 \%$, and $37.544 \%$ after $\mathrm{GO}$ modification. The increase of $\mathrm{C}$, and $\mathrm{O}$, decrease of $\mathrm{N}$ can be attributed to the attachment of $\mathrm{GO}$ on the surface of the CGY. After reduction, the $\mathrm{C}$ increases by $61.855 \%, \mathrm{~N}$ and $\mathrm{O}$ decrease by $2.536 \%$ and $35.609 \%$ respectively. The surface elements change indicates that the reduction process successfully facilitates the transformation of rGO from GO, which is consistent with our previous observation ${ }^{[24]}$.

Figure $2 d-d_{2}$ show the surface morphology of the pristine CGY, GO-CGY-10, and rGO-CGY-10 respectively. Comparing with the pristine CGYs with smooth surfaces, the GO-CGY-10 shows rough and uneven surfaces due to the existence of GO layers, and rGO-CGY-10 has the wrinkled and crumpled surface which is similar to graphene fibers ${ }^{[25-27]}$. This volume shrinkage is mainly due to intermittent pad dyeing and dehydration shrinkage ${ }^{[28]}$. The volume shrinkage is beneficial to compacting the $\mathrm{rGO}$ coatings into a dense structure and forming a strong interaction between the rGO and the pristine CGYs ${ }^{[29]}$. Ascorbic 
acid, as a green reducing agent, promotes the reduction of GO on the surface of the pristine CGY without observable damage to the yarn ${ }^{[30]}$. Figure $2 \mathrm{e}-\mathrm{e}_{2}$, Fig. $2 \mathrm{f}-\mathrm{f}_{2}$, and Fig. $2 \mathrm{~g}-\mathrm{g}_{2}$ show the surface element distribution of the pristine CGY, GO-CGY-10, and rGO-CGY-10 respectively. The surface morphology and element distribution indicate that graphene is uniformly covered onto the CGFs, which is of great significance to both conductivity and stability of the yarn.

Figure 3a shows the Raman spectra of the GO and rGO-modified CGYs. The D-band at $1370 \mathrm{~cm}^{-1}$ (related to defects or heteroatom-doping) and G-band at $1590 \mathrm{~cm}^{-1}$ (attributed to the vibrations of the $\mathrm{sp}^{2}$ bonded crystalline carbon), indicate that graphene wrapped onto the pristine CGYs successfully. The GOCGY shows a higher $D$ band and rGO-CGY shows a higher $G$ band, which can be seen in the highresolution fitting spectrum of Raman spectra of the GO-CGY (Fig. 3b) and the high-resolution fitting spectrum of Raman spectra of the rGO-CGY (Fig. 3c) respectively. The intensity ratio of D-band and Gband increases from 0.9 to 1.2 and the full width at half maximum (FWHM) of the D-band decreases from 71 to 53 (Fig. 3d,). The enhancement of D-band is mainly attributed to the formation of small-size $\mathrm{sp}^{2}$ domains (disordered carbon) during reduction process. The decrease of FWHM suggests an increase in the structural order. These changes show that GO-CGYs are chemically converted into rGO-CGYs, which is consistent with graphene-coated elastic wearable electronics reported in literature ${ }^{[31]}$.

Figure $3 e$ shows changes in surface wettability of the pristine CGYs and the CGYs with graphene coating. The contact angles increase greatly from around $20^{\circ}$ (the pristine CGYs) to about $150^{\circ}$ (the rGO-CGY) after functionalization. With rGO modification for 4 pad dyeing cycles, the contact angle has reached up to $145^{\circ}$. While with the increase of pad dyeing, the contact angle raises slowly up to $150^{\circ}$ after 10 cycles. The increase of the surface contact angle can be attributed that the pristine CGY is hydrophilic material ${ }^{[17]}$, while graphene is the hydrophobic materia| ${ }^{[32]}$. The coating element (i.e., graphene) on the CGYs surface directly contacting water drops leads to higher contact angles. In addition, the surface wettability depends on not only the hydrophilicity and hydrophobicity but also the surface roughness of CGY. The graphene coating can increases the surface roughness of CGY, resulting in high hydrophilicity [33]. Such change of the surface roughness of CGY can be confirmed through surface morphology (Fig. 2 d-d d $_{2}$.

Figure $3 \mathrm{f}$ shows the thermal stability of the CGYs. With the increase in temperature, the mass of CGYs becomes lighter. When the temperature rises from $20^{\circ} \mathrm{C}$ to $700^{\circ} \mathrm{C}$, the corresponding weight loss reaches about $85 \%$. The masses of CGYs only have a little loss at $100^{\circ} \mathrm{C}$, which is mainly due to the loss of moisture. At around $400{ }^{\circ} \mathrm{C}$, rGO begins to be decomposed, the rGO-CGY-10 shows a more obvious weight loss. The loss can be attributed to the thermal-induced destroy in CGYs, as graphene has remarkable temperature stability and tolerance. It also proves that increasing dyeing cycles can increase the graphene amount on the CGYs, thus enhance their conductivity.

\subsection{Hydrophilicity and mechanical properties}


The typical tensile strain curves of pristine and rGO-modified CGYs in Fig. 4a exhibit that graphene coating can result in a higher breaking strength and a shorter shifting distance as well as a higher modulus. The breaking strength of the pristine CGYs is about $168 \mathrm{cN}$, and it will gradually increase with the dyeing times (Fig. 4b). After 10-cycle dyeing, the breaking strength finally becomes $195 \mathrm{cN}$. The growth in breaking strength is due to the attachment of graphene on the fiber surface, promoting a stronger combination among fibers ${ }^{[34]}$. Figure $4 \mathrm{c}$ shows that the increase in dyeing cycles slightly decreases the tensile strain of the CGYs, of which tensile strain is $4.1 \%$ and $7.2 \%$ for pristine CGYs and the rGO-CGY-10 respectively.

Figure $4 \mathrm{~d}$ shows the electrical resistance of CGYs after graphene coating. The electrical resistance of the rGO-CGY drastically decreases with pad dyeing cycles, from about $800 \mathrm{k} \Omega$ for rGO-CGY-4 to $210 \mathrm{k} \Omega$ for rGO-CGY-10. We further calculate the electrical conductivity of the rGO-CGYs (Fig. 4e). Compared with the electrical conductivity of rGO-CGY-4, the electrical conductivity of rGO-CGY-10 is obviously improved. The rGO-CGY-10 exhibits high electrical conductivity of $6.9 \mathrm{~S} / \mathrm{m}$, while the rGO-CGY-4 only presents a volume conductivity of $2 \mathrm{~S} / \mathrm{m}$. With the combination of graphene, the strong $\pi-\pi$ interactions between adjacent graphene form interconnected electron-transport pathways. With more pad dyeing cycles, rGO-CGY-10 holds more electron-transport pathways and obtains high electrical conductivity.

The range analysis of the coating parameters in determining the electrical conductivity of rGO-CGYs in Fig. $4 \mathrm{f}$ shows that range value of the additive concentration of $\mathrm{GO}$ solution, the reduction time, and the pad dyeing cycles is $3.665,1.344$, and 2.048 respectively. It exhibits that the electrical conductivity of rGoCGYs largely depends on the additive concentration of GO solution and pad dyeing cycles, and the reduction time has little effect on it. When the concentration of $\mathrm{GO}$ solution is $3 \mathrm{mg} / \mathrm{mL}$, the content of graphene is low, while the concentration of GO solution is $7 \mathrm{mg} / \mathrm{mL}$, the viscosity of graphene solution is too high to adhere to the surface of CGY. Both of them lead to fewer electron-transport pathways. The degree of graphene coating on yarns' surface can be augmented with the increase of pad dyeing cycles, which also can raise the amounts of electron-transport pathways on the surface of CGYs.

Figure 5a presents the FTIR spectrum of the pristine CGY, GO-CGY-10, and rGO-CGY-10. The FTIR spectrum of the pristine CGY displays peaks around 3340, 2900, 1645, 1430 and $1060 \mathrm{~cm}^{-1}$, which are mainly attributed to the $\mathrm{OH}$ stretching, asymmetrical $\mathrm{C}-\mathrm{H}$ stretching, $\mathrm{C}=\mathrm{O}$ stretching, $\mathrm{CH}_{2}$ symmetrical bending and $\mathrm{C}-\mathrm{O}$ stretching respectively ${ }^{[35]}$. After graphene coating, no evident change is found in the FTIR spectrum of GO-CGY-10, which is consistent with the previous research [35]. Whereas, the FTIR spectrum of rGO-CGY-10 changed obviously after the treatment of ascorbic acid. The peak at $1645 \mathrm{~cm}^{-1}$ disappears and the peak at 2900 and $3340 \mathrm{~cm}^{-1}$ become weaker, which implies that $\mathrm{GO}$ on the surface of CGY is successfully reduced to $\mathrm{rGO}$ after the treatment of ascorbic acid ${ }^{[35]}$.

Figure $5 \mathrm{~b}$ shows the characteristic absorption wavenumbers of intramolecular hydrogen bond $\mathrm{O}(2) \mathrm{H} \cdots \mathrm{O}(6), \mathrm{O}(3) \cdots \mathrm{O}(5)$, and $\mathrm{O}(6) \cdots \mathrm{O}\left(3^{\prime}\right)$ in CGYs are $3310-3233 \mathrm{~cm}^{-1}, 3375-3340 \mathrm{~cm}^{-1}$, and $3455-$ $3410 \mathrm{~cm}^{-1}$, which are named $\otimes, \otimes$, and $\otimes$ respectively ${ }^{[36]}$. The percentage of $\otimes$ changes slightly from 61.32 


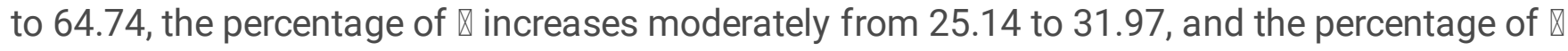
decreases obviously from 13.54 to 3.29. It reveals that the content of $\triangle$ in CGY decreases during the treatment. CGYs consisting of celluloses are rich in positively charged hydrogen, while GO has plenty of negative groups such as carboxyl and hydroxyl groups ${ }^{[36,37]}$. We postulate a bonding mechanism between CGYs and rGO, which is shown in Fig. 5c. The pristine CGY is coated with $\mathrm{GO}$ and using ascorbic acid to convert GO into rGO. The negatively charged GO species and positively charged hydrogen in CGY are combined by electrostatic attraction, which is consistent with reported work ${ }^{[38]}$. Such postulation can also be supported by the FTIR and EDS results. The atomic percentage of $O$ not only does not increase obviously after $\mathrm{GO}$ coating but also decreases significantly after reduction. Meanwhile, the content of the $\triangle$ in CGYs greatly drops during the whole process.

To evaluate the washability of the graphene-functionalized CGY, we wash the rGO-CGY-10 with deionized water and laundry liquid. With the washing in deionized water, the volume conductivity of the rGO-CGY-10 slightly decreases with the increase of washing time (Fig. 6a). Similarly, the volume conductivity shows the same trend decrease when the yarn is washed in laundry liquid (Fig. 6b). The electrostatic attraction between negatively charged GO species and positively charged hydrogen in CGY enhances the bonding. Notably, the washed yarns still maintain very good conductivity after washing in both deionized water and laundry liquid, showing the good stability of the graphene coating.

Figure $6 \mathrm{c}-\mathrm{C}_{2}$ show the photos of the rGO-CGY-10 with bending (0-75\%). Figure $6 \mathrm{~d}$ and Fig. 6e present the relative resistance change of the $\mathrm{rGO}-\mathrm{CGY}-10$ with the bending of $50 \%$ and $75 \%$ respectively. The bending was calculated by distance change/initial length of CGYs during the bending test. We show the resistance change with $\left(R-R_{0}\right) / R_{0}$, where the $R_{0}$ and $R$ refer to the initial resistance and the resistance under bending respectively. The resistance of the yarn slightly increases from 255 to $262 \mathrm{k} \Omega$ with the bending ranging from 0 to $50 \%$, and this trend is very stable throughout the cyclic bending process. However, the resistance change is as low as 3.2\%. Even under high-degree bending up to $75 \%$, the resistance change is still very minor (4.3\%). The CGY has a very limited response to bending, which means that the graphene-coated CGYs have a certain anti-interference performance to bending and show great potential in flexible wearable electronic devices.

\section{Conclusion}

In a summary, we have reported a conductive CGY through pad dyeing with graphene, showing mass production and high performance in conductivity. When GO concentration and pad dyeing cycles reach 5 $\mathrm{mg} / \mathrm{mL}$ and 10 times respectively, the graphene-coated CGYs exhibit high electrical conductivity and better breaking strength. The range analysis shows that electrical conductivity of the graphene-coated CGYs exhibit strong dependence on both the concentration of graphene oxide and dyeing cycles, and very marginal dependence on reduction time. This coating approach promotes the formation of hydrogenoxygen bond between CGFs and graphene, endowing the functionalized CGYs with good conductive stability to not only washing but also bending. Particularly, the pad dyeing for preparing conductive CGYs 
can be rapidly extended to almost any other yarns, paving a new way for developing large-scale production, low-cost, and high-performance conductive yarns.

\section{Declarations}

\section{Acknowledgements}

This work was financially supported by National Key R\&D Program of China (2021YFE0111100) and Shanghai Science and Technology Committee (21015800600) and the "Top six talent peaks" program of Jiangsu (GDZB-035) and Science and Technology Project of Nantong (JC2020082). We would like to thank for the support of China National Textile and Apparel Council (J202002), Nature Science Foundation of Jiangsu (BK20191191) and joint scientific research project of Sino-foreign cooperative education platform of Jiangsu Higher Education Institutions (5011500720) and projects with numbers FZ20190257 and $2021 f \times 010104$.

\section{Disclosure}

The authors have nothing to disclose regarding the conflict of interest.

\section{References}

1. Liu ZK, Zheng Y, Jin L, Chen KL et al., (2021) Highly Breathable and Stretchable Strain Sensors with Insensitive Response to Pressure and Bending. Advanced Functional Materials. 31(14)

2. Lu Y, Sun H, Cheng JY, Myong J et al (2020) High performance flexible wearable strain sensor based on rGO and AgNWs decorated PBT melt-blown non-woven fabrics. Sensors and Actuators a-Physical, p 315

3. Gandla S, Naqi M, Lee MG, Lee JJ et al., (2020) Highly Linear and Stable Flexible Temperature Sensors Based on Laser-Induced Carbonization of Polyimide Substrates for Personal Mobile Monitoring. Advanced Materials Technologies. 5(7)

4. Wang YM, Zhang LW, Zhang ZW, Sun PY et al (2020) High-Sensitivity Wearable and Flexible Humidity Sensor Based on Graphene Oxide/Non-Woven Fabric for Respiration Monitoring. Langmuir 36(32):9443-9448

5. Liu Z, Li Z, Zhai H, Jin L et al (2021) A highly sensitive stretchable strain sensor based on multifunctionalized fabric for respiration monitoring and identification. Chemical Engineering Journal

6. Zhao Y, Ren MN, Shang Y, Li JN et al (2020) Ultra-sensitive and durable strain sensor with sandwich structure and excellent anti-interference ability for wearable electronic skins. Composites Science and Technology, p 200

7. Liu ZK, Chen KL, Fernando A, Gao Y et al., (2021) Permeable graphited hemp fabrics-based, wearingcomfortable pressure sensors for monitoring human activities. Chemical Engineering Journal. 403 
8. Won C, Lee S, Jung HH, Woo J et al (2020) Ultrasensitive and Stretchable Conductive Fibers Using Percolated Pd Nanoparticle Networks for Multisensing Wearable Electronics: Crack-Based Strain and H-2 Sensors. Acs Applied Materials Interfaces 12(40):45243-45253

9. Ren F, Song DP, Li Z, Jia LC et al (2018) Synergistic effect of graphene nanosheets and carbonyl ironnickel alloy hybrid filler on electromagnetic interference shielding and thermal conductivity of cyanate ester composites. Journal of Materials Chemistry C 6(6):1476-1486

10. Ozen MS, Sancak E, Soin N, Shah TH et al (2018) Unprecedented Electromagnetic Shielding Effectiveness of Lightweight Nonwoven Ag/PA66 Fabrics. Fibers Polymers 19(2):321-330

11. Jia LC, Xu L, Ren F, Ren PG et al (2019) Stretchable and durable conductive fabric for ultrahigh performance electromagnetic interference shielding. Carbon 144:101-108

12. Lin ZM, Yang J, Li XS, Wu YF et al., (2018) Large-Scale and Washable Smart Textiles Based on Triboelectric Nanogenerator Arrays for Self-Powered Sleeping Monitoring. Advanced Functional Materials. 28(1)

13. Geim AK, Novoselov KS (2007) The rise of graphene. Nature Materials 6(3):183-191

14. Molina J (2016) Graphene-based fabrics and their applications: a review. Rsc Advances 6(72):68261-68291

15. Balandin AA, Ghosh S, Bao WZ, Calizo I et al (2008) Superior thermal conductivity of single-layer graphene. Nano Lett 8(3):902-907

16. Zhao ZY, Zheng ZZ, Chen P, Zhang HF et al (2019) Pre-treatment of Calotropis gigantea fibers with functional plasticizing and toughening auxiliary agents. Textile Research Journal 89(19-20):39974006

17. Zheng Y, Cao EJ, Tu LX, Wang AQ et al (2017) A comparative study for oil-absorbing performance of octadecyltrichlorosilane treated Calotropis gigantea fiber and kapok fiber. Cellulose 24(2):989-1000

18. Chen Q, Zhao T, Wang M, Wang J (2013) Studies of the fibre structure and dyeing properties of Calotropis gigantea, kapok and cotton fibres. Color Technol 129(6):448-453

19. Ganeshan P, Kumaran SS, Raja K, Venkateswarlu D (2018) An investigation of mechanical properties of madar fiber reinforced polyester composites for various fiber length and fiber content. Materials Research Express. 6(1)

20. Dwivedi UK, Singh S, Shukla SC, Hashmi SA, R (2021) Investigation on tribo-characteristics of Calotropis gigantea fiber-reinforced-CNT modified polymer composites. Polymer Bulletin

21. Xu LL, Liu ZK, Zhai H, Chen X et al (2020) Moisture-Resilient Graphene-Dyed Wool Fabric for Strain Sensing. Acs Applied Materials Interfaces 12(11):13265-13274

22. Cho SY, Yun YS, Lee S, Jang D et al (2015) Carbonization of a stable beta-sheet-rich silk protein into a pseudographitic pyroprotein. Nat Commun 6:7145

23. Kim J, Cote LJ, Huang JX (2012) Two Dimensional Soft Material: New Faces of Graphene Oxide. Acc Chem Res 45(8):1356-1364 
24. Cai GM, Xu ZL, Yang MY, Tang B et al (2017) Functionalization of cotton fabrics through thermal reduction of graphene oxide. Appl Surf Sci 393:441-448

25. Ding XT, Bai J, Xu T, Li CX et al (2016) A novel nitrogen-doped graphene fiber microelectrode with ultrahigh sensitivity for the detection of dopamine. Electrochemistry Communications 72:122-125

26. Abdou JP, Reynolds KJ, Pfau MR, van Staden J et al (2016) Interfacial crystallization of isotactic polypropylene surrounding macroscopic carbon nanotube and graphene fibers. Polymer 91:136-145

27. Sheng LZ, Wei T, Liang Y, Jiang LL et al (2017) Ultra-high toughness all graphene fibers derived from synergetic effect of interconnected graphene ribbons and graphene sheets. Carbon 120:17-22

28. Chen SH, Ma WJ, Cheng YH, Weng Z et al (2015) Scalable non-liquid-crystal spinning of locally aligned graphene fibers for high-performance wearable supercapacitors. Nano Energy 15:642-653

29. Park SK, Seong CY, Piao Y (2015) A Simple Dip-coating Approach for Preparation of Threedimensional Multilayered Graphene-Metal Oxides Hybrid Nanostructures as High Performance Lithium-Ion Battery Electrodes. Electrochimica Acta 176:1182-1190

30. Zhang JL, Yang HJ, Shen GX, Cheng P et al (2010) Reduction of graphene oxide via L-ascorbic acid. Chemical Communications 46(7):1112-1114

31. Wang CY, Zhang MC, Xia KL, Gong XQ et al (2017) Intrinsically Stretchable and Conductive Textile by a Scalable Process for Elastic Wearable Electronics. Acs Applied Materials Interfaces 9(15):1333113338

32. Zhang GJ, Guo XX, Wang SL, Wang XL et al (2014) New graphene fiber coating for volatile organic compounds analysis. Journal of Chromatography B-Analytical Technologies in the Biomedical Life Sciences 969:128-131

33. Chen PY, Sodhi J, Qiu Y, Valentin TM et al (2016) Multiscale Graphene Topographies Programmed by Sequential Mechanical Deformation. Adv Mater 28(18):3564-3564+

34. Mackin TJ, Warren PD, Evans AG (1992) Effects of Fiber Roughness on Interface Sliding in Composites. Acta Metallurgica Et Materialia 40(6):1251-1257

35. Xu LL, Guo MX, Liu S, Bian SW (2015) Graphene/cotton composite fabrics as flexible electrode materials for electrochemical capacitors. Rsc Advances 5(32):25244-25249

36. Oh SY, Yoo DI, Shin Y, Kim HC et al (2005) Crystalline structure analysis of cellulose treated with sodium hydroxide and carbon dioxide by means of X-ray diffraction and FTIR spectroscopy. Carbohydrate Research 340(15):2376-2391

37. Bunshi SUNL, and FUGETSU (2000) Massive production of graphene oxide from expanded graphite 38. Dashairya L, Rout M, Saha P (2018) Reduced graphene oxide-coated cotton as an efficient absorbent in oil-water separation. Advanced Composites Hybrid Materials 1(1):135-148

\section{Figures}




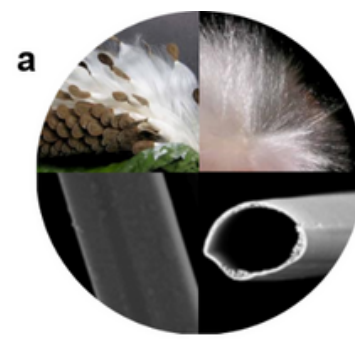

Calotropis gigantea fibers

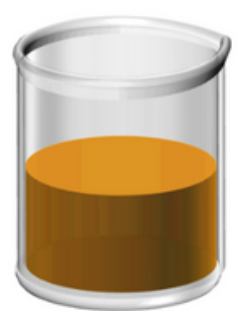

Graphene oxide solution

b
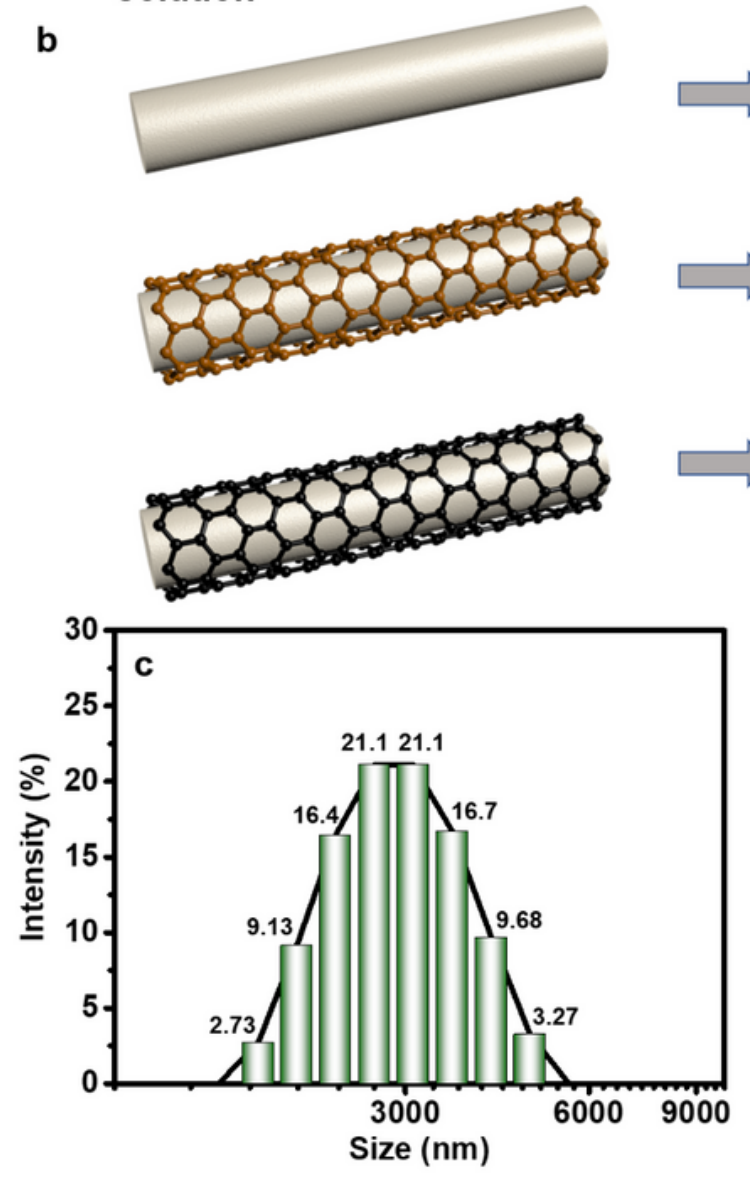$$
\text { b }
$$

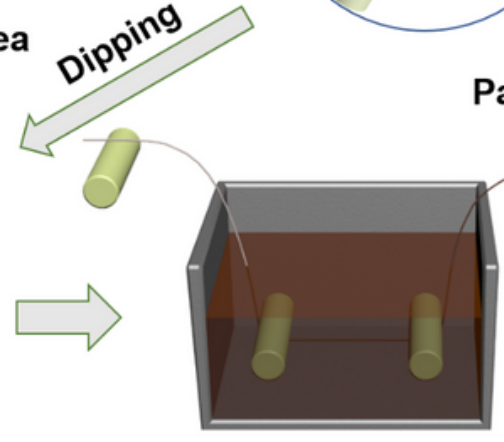

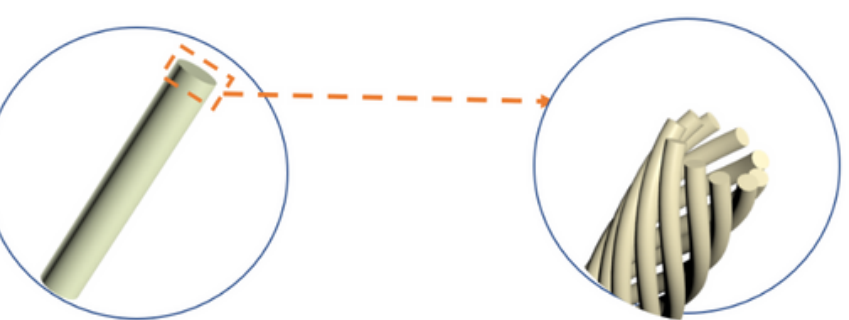

Pad dyeing

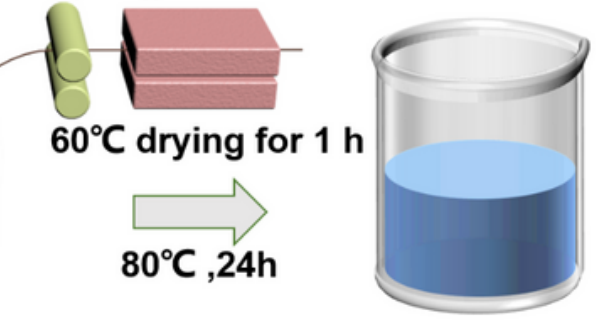

Ascorbic acid
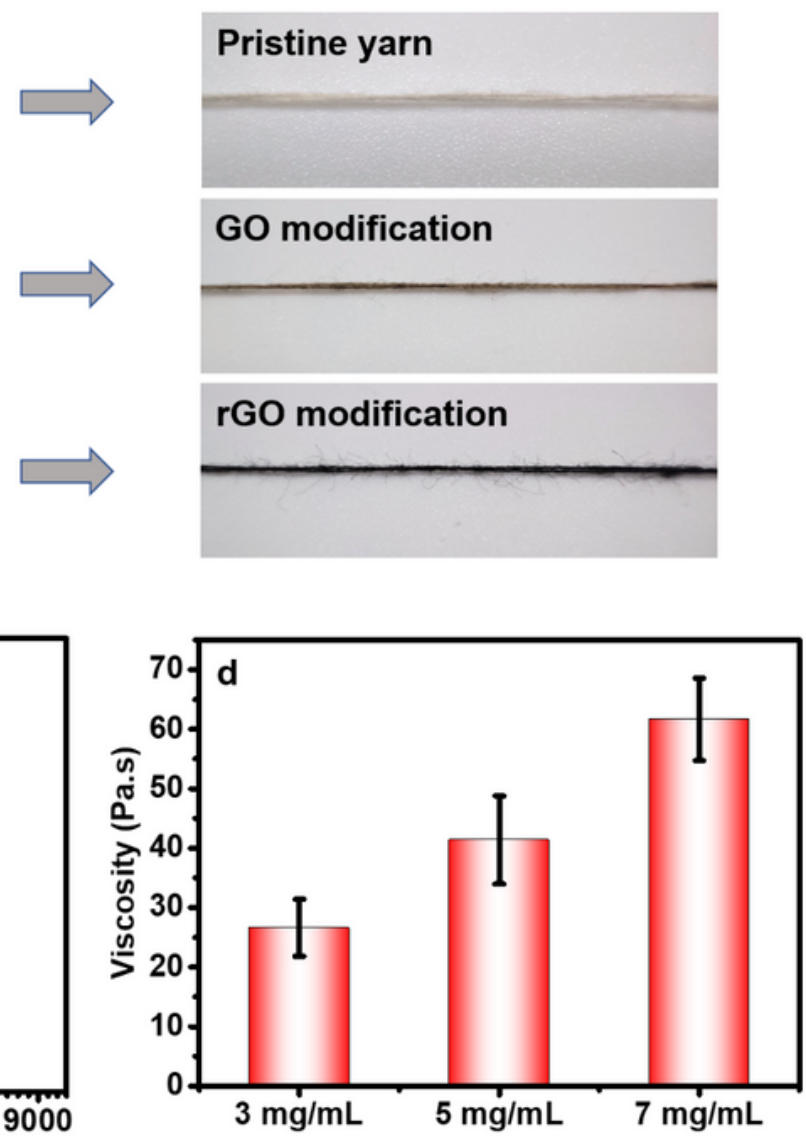

Figure 1

(a) Schematic diagram of GO-CGYs through pad dyeing. (b) The illustrations and photos of the pristine, GO-modified, and rGO-modified CGYs. (c) The particle size distribution of the GO solution, (d) the viscosity of GO solution. 


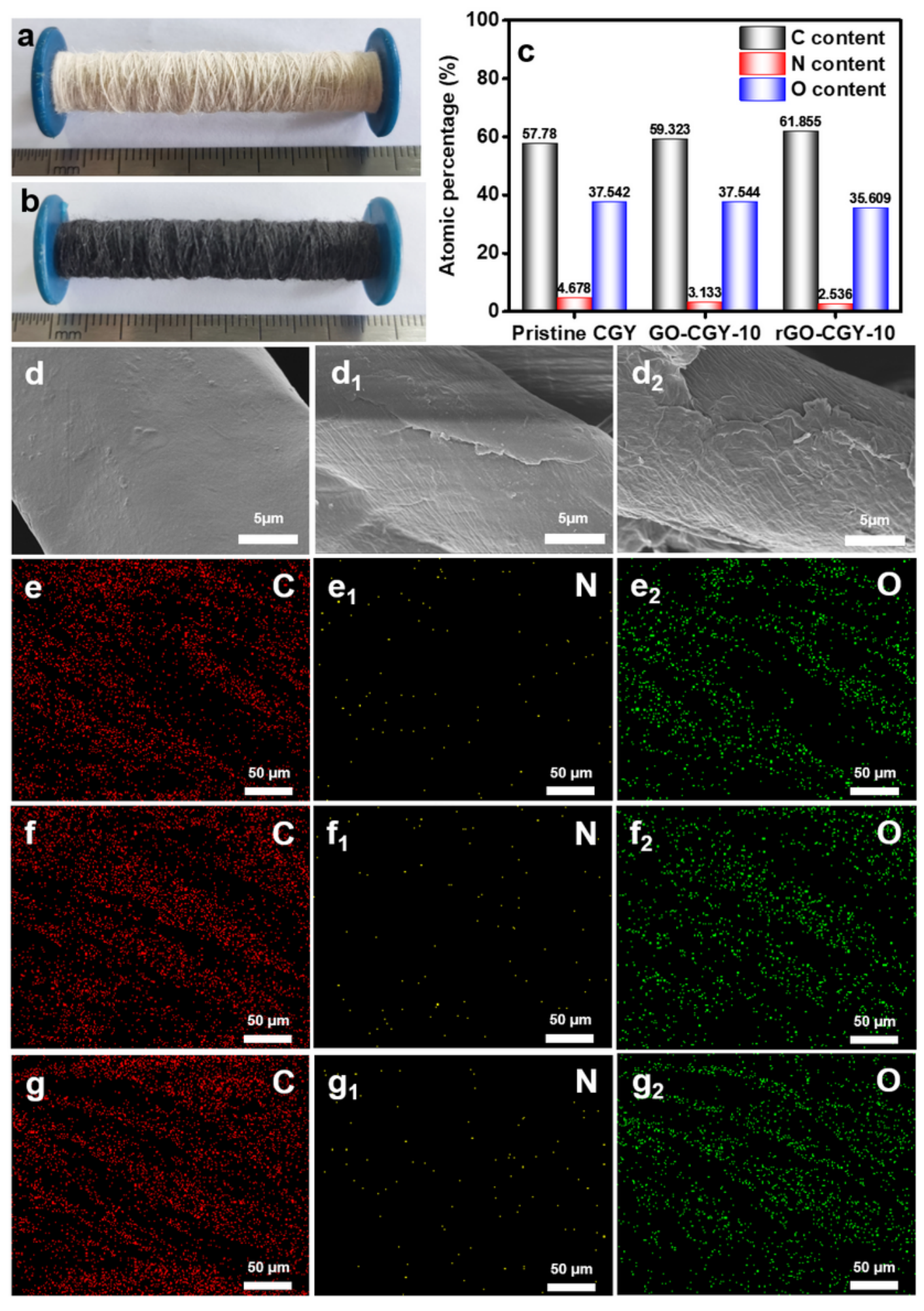

Figure 2

(a) The photos of the pristine CGYs. (b) The photos of the rGO-CGY-10. (c) The surface element content of CGYs, the surface morphology of (d) the pristine CGY, (d1) the GO-CGY-10, (d2) the rGO-CGY-10, (e-e2) $\mathrm{C} / \mathrm{N} / \mathrm{O}$ distributions of the pristine CGY, (f-f2) C/N/O distributions of the GO-CGY-10, (g-g2) C/N/O distributions of the rGO-CGY-10. 

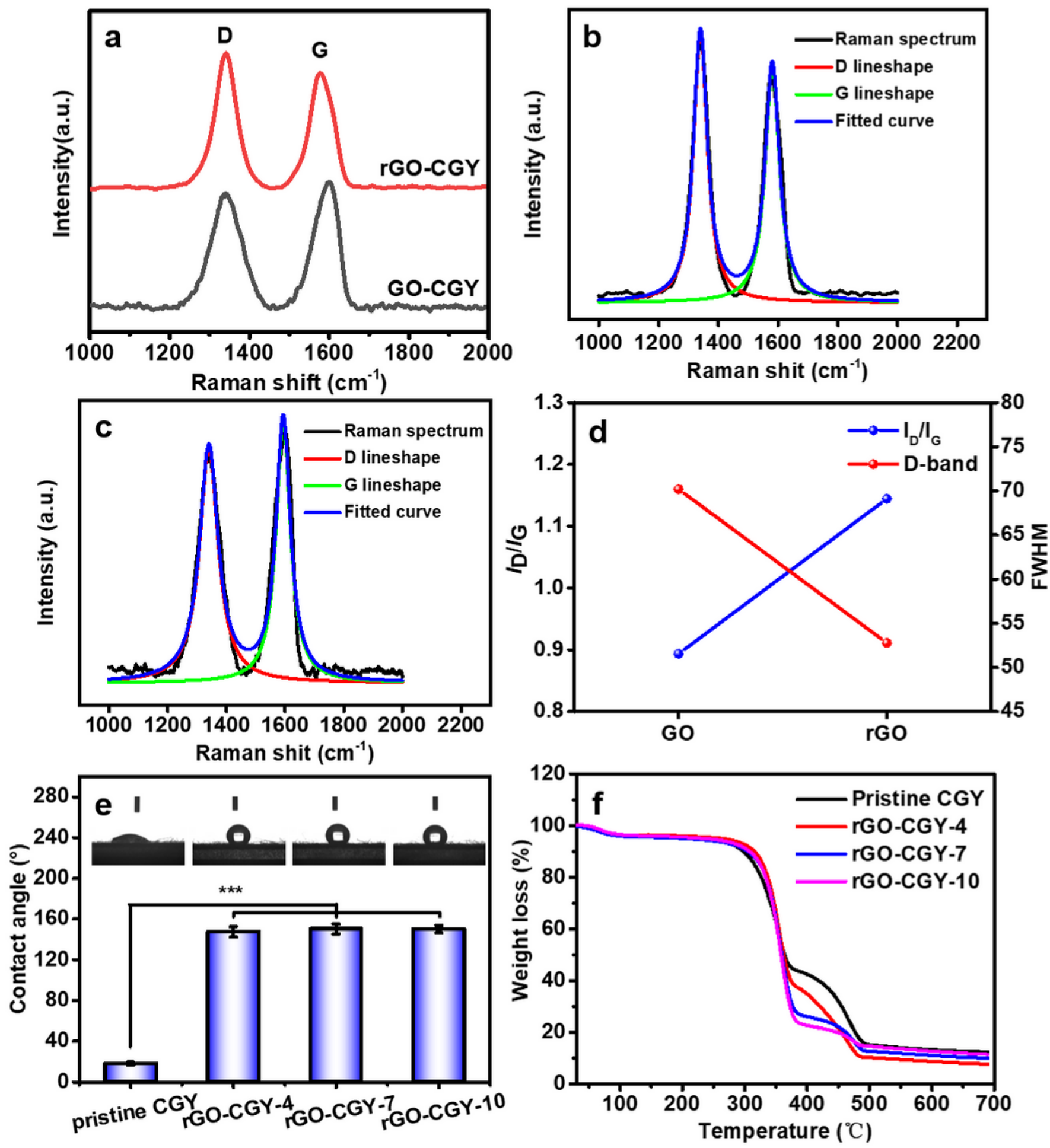

Figure 3

(a) Raman spectra of the CGYs after the functionalization. (b) High-resolution spectrum of Raman spectra of the GO-CGY-10. (c) High-resolution spectrum of Raman spectra of the rGO-CGY-10. (d) The intensity ratios of D-band and G-band and the full width at half maximum (FWHM) of the D-band for the GO-CGY-10 and rGO-CGY-10. (e) The contact angle of the pristine CGYs after graphene coating. (f) The TGA curves of graphene-coated CGYs. 

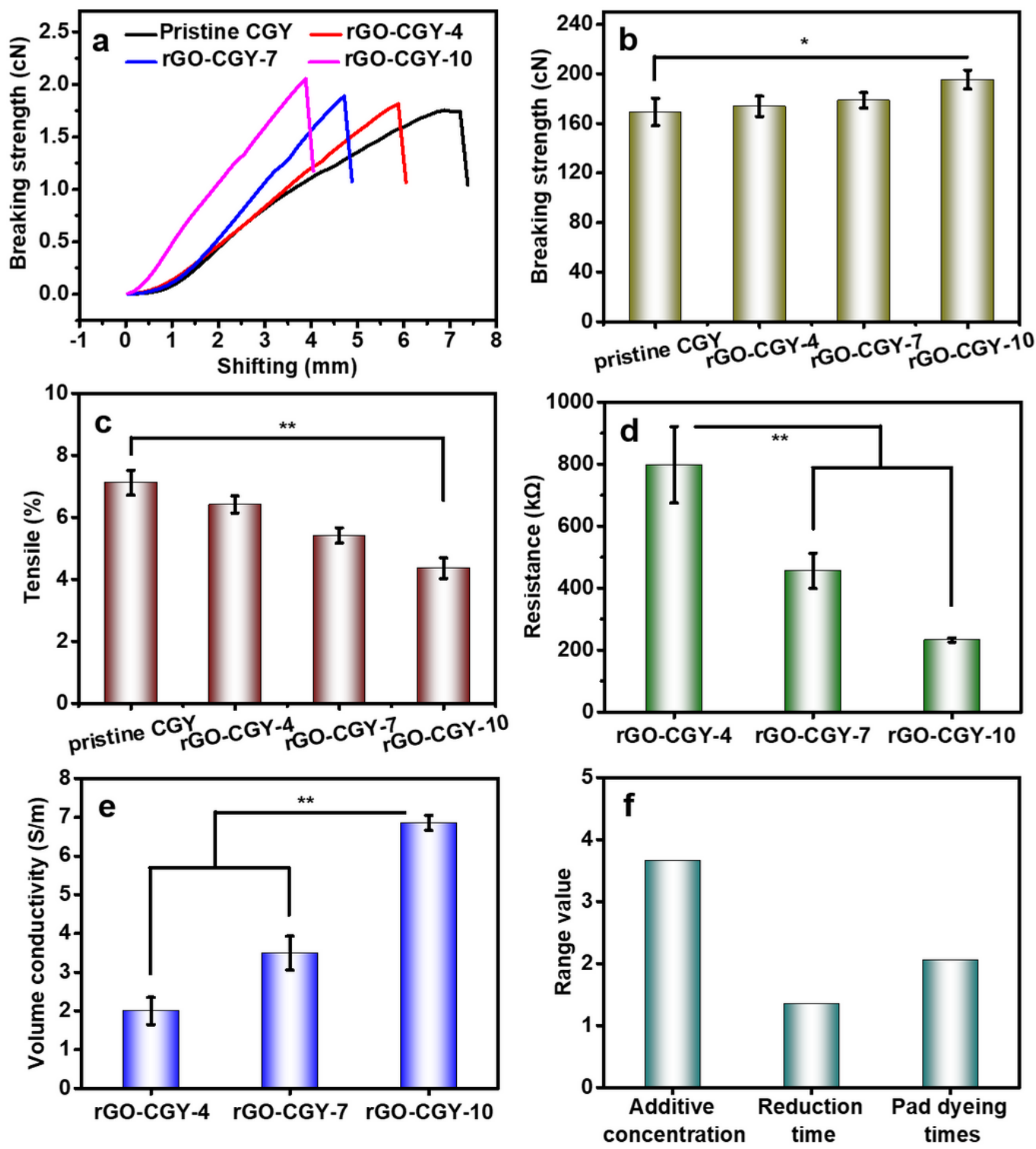

Figure 4

(a) The stretch curve of CGYs. (b) The breaking strength of the graphene-coated CGYs. (c) The tensile strain of the graphene-coated CGYs. (d) The electrical resistance of the rGO-CGYs. (e) The volume electrical conductivity of the rGO-CGYs. (f) A range analysis of the electrical conductivity of the rGOCGYs. 

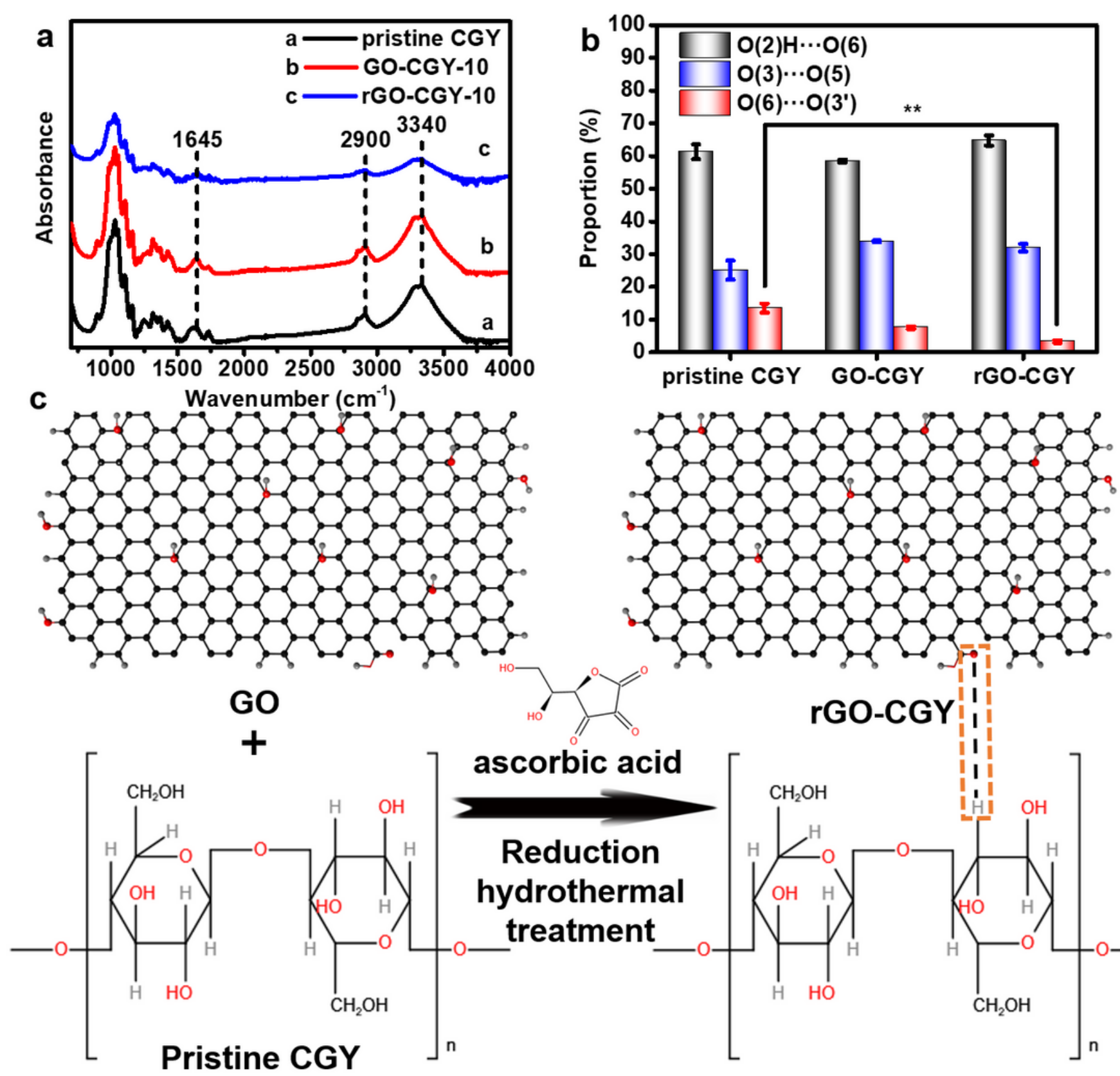

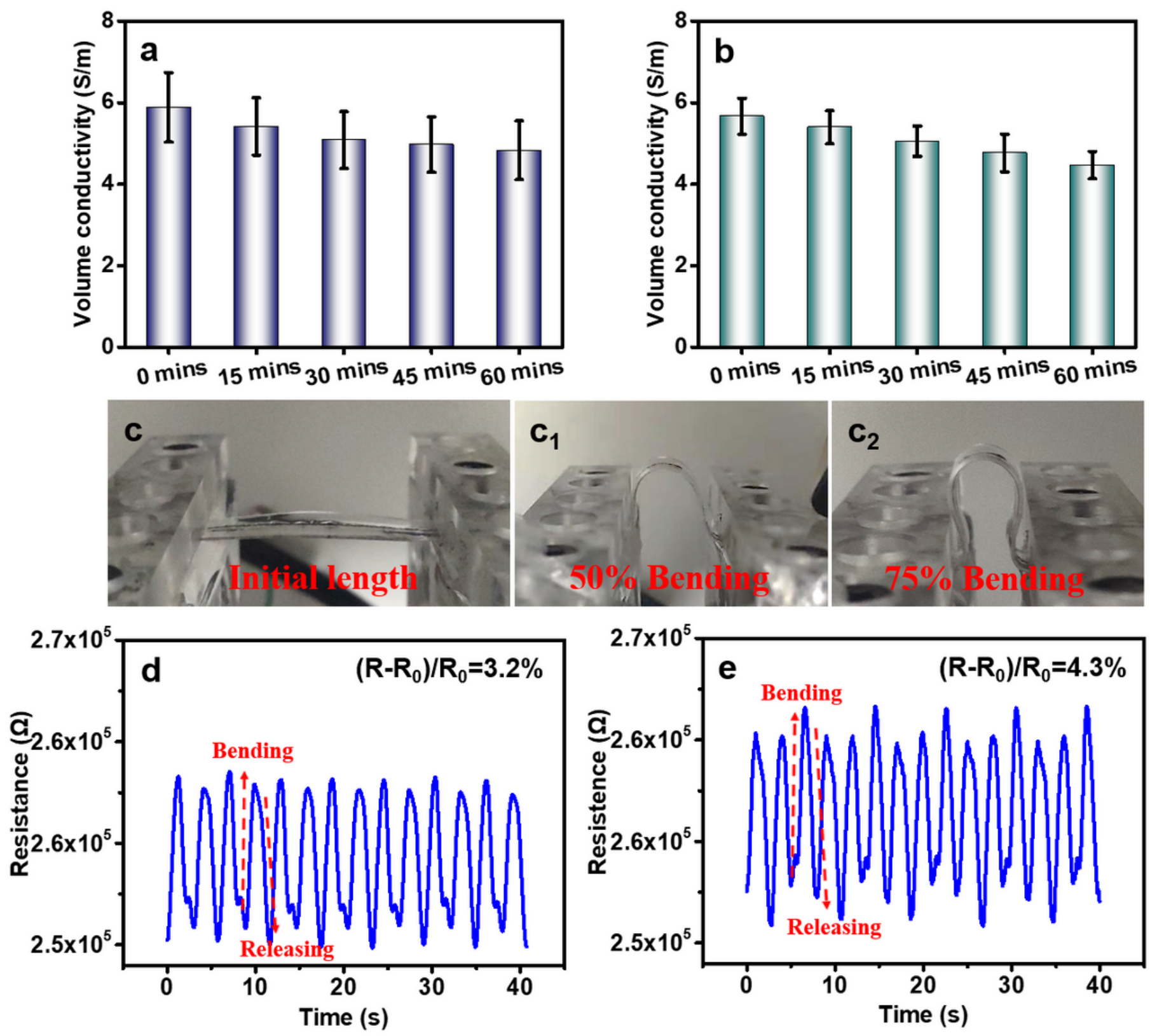

Figure 6

(a) The volume electrical conductivity changes of the rGO-CGY-10 with deionized water washing. (b) The volume electrical conductivity changes of the rGO-CGY-10 with laundry liquid washing. (c-c2) The photos of rGO-CGY-10 with initial length, $50 \%$ bending, and $75 \%$ bending. (d) The relative resistance changes and image of the rGO-CGY- 10 with $50 \%$ bending. (e) The relative resistance changes and image of the rGOCGY-10 with $75 \%$ bending. 